\title{
THE VARIATION WITH ANGLE OF EMISSION OF THE RADIATION FROM METALS BOMBARDED BY SLOW ELECTRONS
}

\author{
By C. Boeckner
}

ABSTRACT

The radiation from $\mathrm{Pt}$ and $\mathrm{W}$, with its electric vector parallel to the plane of emission, increases rapidly with increasing emission angle. For the other direction of polarization the radiation varies only slowly with angle. The variation is given by the function $\left(1-R_{\theta}\right) / \cos \theta$ where $R_{\theta}$ is the reflection coefficient of the metal for light incident at angle $\theta$. A discussion of the variation of emission from surface layers with angle is given, based on T. C. Fry's theory of lamellar absorption. The disagreement with the observed variation is in the direction to be accounted for if the radiation is initially emitted anisotropically (in a manner similar to the continuous $\mathrm{X}$ rays) and is later modified by refraction effects.

It is shown that the angle variation is somewhat similar to that expected for the inverse vectorial photo-electric effect.

\section{CONTENTS}

I. Introduction

II. Method... 584

III. Results

IV. Discussion

1. Radiation from a thin layer 586

2 . The intensity variation of thermal radiation with angle of emission

3. Surface emission and "lamellar absorption"

4. Relation to the inverse photo-electric effect._._._._._. 589

5. Relation to the variation of intensity with angle of the

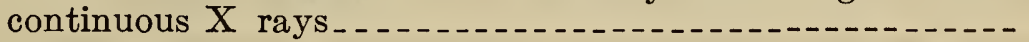

V. Summary

\section{INTRODUCTION}

There is considerable evidence showing that metals when bombarded by electrons emit visible and ultra-violet radiation. In 1920 Lillienfeld ${ }^{1}$ reported a blue gray luminescence appearing at the focal spot of an X-ray tube anticathode, due apparently to the excitation of the metal atoms by the high speed cathode rays. A similar radiation was reported by Foote, Meggers, and Chenault ${ }^{2}$ as emanating from a target bombarded by somewhat slower electrons having energies of the order of 1,000 volts.

Recently F. L. Mohler ${ }^{3}$ and the author have described a method for studying the radiation from metals bombarded by very slow electrons having energies of from 2 to 15 volts. The method makes use of the fact that a small metal electrode in a gas discharge draws very intense electron currents when maintained at a potential positive to the surrounding space. It was found that with the large current densities thus obtained ( 4 or $\left.5 \mathrm{amp} . \mathrm{cm}^{5}\right)$, metals emitted radiation

1 Lillienfeld and Rother, Phys. Zeits., vol. 21, p. 49, 1920.

2 Foote, Meggers, and Chenault, J. Opt. Soc. Am., vol. 9, p. 541, 1924.

8 F. L. Mohler and C. Boeckner, B. S. Jour. Research, vol. 6 (RP297), p. 673, 1931. F. L. Mohler, B. S. Jour. Research, vol. 8 (R P421), p. 357, 1932. 
having a continuous spectrum of rather uniform intensity in the visible and ultra-violet.

Some of the characteristics of this radiation are the approximate equality of the absolute intensities from most metals; the exceptional behavior of silver in possessing a very intense emission band in the ultra-violet; and the fact that at low electron velocities the spectrum possesses a high frequency limit in the ultra-violet. It may also be mentioned that the radiation is thought to be the analogue of the continuous X-ray spectrum since the absolute intensity is of the order predicted for the "bremsstrahlung" produced by slow electrons.

The present paper deals with the polarization of the radiation and the variation of intensity with the angle of emission. The measurements were undertaken with the thought that the intensity-angle relation might be similar to that of the continuous $\mathrm{X}$ rays. It will be shown later that the velocity of the electrons is normal to the metal surface; it might therefore be expected as in the case of the continuous $\mathrm{X}$ rays that the radiation would be most intense when observed in a direction perpendicular to the direction of motion of the electrons. By analogy the radiation might also be expected to be polarized with the electric vector parallel to this direction.

\section{METHOD}

To study the variation of intensity with angle, a cylindrical electrode was observed in a direction perpendicular to its axis. An enlarged image of the cylinder was focused upon the spectograph slit, the axis of the image being in the plane of the slit and perpendicular to it. From a study of the variation of intensity across the image, information could be deduced concerning the variation of intensity with emission angle. It is clear for example that the radiation forming the edges of the image is emitted at a grazing angle while light at the center is emitted normal to the cylinder surface.

It was thought desirable to study the intensity variation for both the significant directions of polarization of the radiation. A quartz double image prism was therefore placed in the optical train so that one of the images had the electric vector of its radiation parallel to the plane of emission (perpendicular to the cylinder axis) and the other perpendicular to the plane. Since the ultra-violet was found to be the most convenient spectral region for the study of the radiation, quartz fluorite achromatic lenses were used to form the cylinder image upon the slit. It was found necessary to place the double image prism on the source side of the lenses to avoid initial depolarization of the radiation by their quartz components.

A discharge in cæsium vapor (several thousandths millimeter pressure) was used as a source of current to the electrodes for numerous experimental reasons. The cylindrical electrode was placed in the positive column of the discharge coaxial with the cylindrical discharge tube. This arrangement was necessary to secure uniform current density over the electrode surface. The measurements were all made with energies of the bombarding electrons of about 7 volts.

It is of interest to mention that the potential drop between the electrode and the discharge occurs in a small sheath several hundredths of a millimeter thick; the electric force is consequently normal to the metal surface. The direction of motion of the electrons is therefore also perpendicular to the metal surface, since their initial energy 
in the discharge is small compared to the potential drop across the sheath.

The metals studied were platinum, tungsten, and silver. The first two metals could be raised to a bright red heat and outgassed by drawing large discharge currents to them while the discharge tube was connected to the pumps. The surfaces were further cleaned by intense ion bombardment.

It was thought of some significance to compare the variation of intensity with angle for a matte surface and a polished surface. A very good diffusely reflecting surface of platinum was prepared by electroplating a layer of platinum black upon a platinum rod and heating to redness in a flame. An attempt was made to prepare a similar tungsten surface by etching a tungsten rod. In this case, however, the surface was far from being a diffuse reflector and showed marked polarization of light reflected from it.

Densitometer records of the blackening across the cylinder image on the spectograms were made by means of a Kip \& Zonen registering microphotometer. The intensities were deduced from these records. A series of differently timed exposures on each spectogram served to give the blackening intensity characteristic of the plate. The wavelength range covered was from 4,200 to about $3,100 \mathrm{~A}$.

The method described has the advantage that the intensities for a range of angles may be obtained from one exposure. It has the disadvantage, however, that the radiation emitted at angles greater than $60^{\circ}$ to the surface normal correspond to a region so near the edge of the image that results are falsified by errors in focus. The method was, however, found to be convenient and was thought to be adequate for at least a rough survey of the intensity relations. Due to the long exposure times (six hours with the double image prism in place) a more direct method would have been tedious. One set of measurements, however, was made of the intensity of emission from platinum at $75^{\circ}$ by comparing the intensity from a large plane electrode observed at an oblique angle with that obtained when observed in a direction perpendicular to the surface.

\section{RESULTS}

For both platinum and tungsten it was found that the radiation with the electric vector parallel to the plane of emission increased rapidly with the angle of emission (the angle between the emitted ray and the normal to the surface). For the other direction of polarization the intensity was constant across the image. Figure 1 shows a plot of the intensity variation across the tungsten cylinder image, the tungsten being bombarded by 7 -volt electrons. The tungsten in this case had been etched in an attempt to render the surface a diffuse reflector. A polished surface, however, gave the same results showing that the intensity is not sensitive to the degree of polish of the surface. There was no appreciable change in variation with angle between 4,200 and $3,100 \mathrm{~A}$.

By drawing large currents to the cylinder, it was possible to heat it to incandescence, enabling measurements to be made of the variation of the intensity of the thermal radiation with angle. For comparison the results are shown on the lower part of the figure.

Figure 2 shows a plot of the intensity-angle variation for polished platinum (full line). The measurements at $75^{\circ}$ were obtained from a 
large plane electrode which could be observed normally and at an oblique angle. It is seen that in this case the variation with angle changes with wave length. In contrast with tungsten the radiation from the matte platinum surface showed no variation with angle. This is perhaps explained by the fact that the platinum surface is more nearly an ideal diffuse reflector.

Silver showed no variation in intensity across the image of the cylinder. This may, however, be explained by the fact that it was

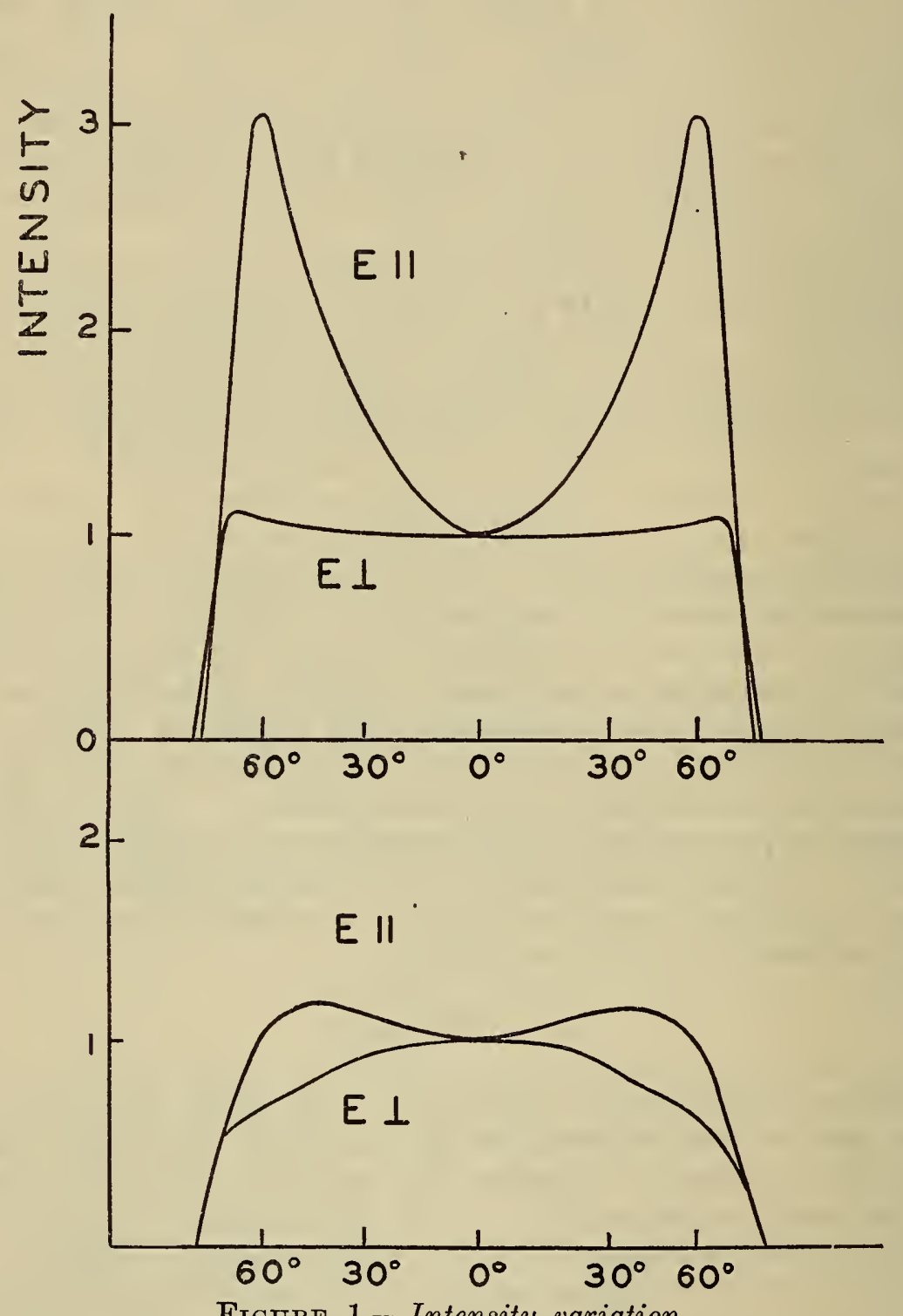

FIGURE 1.--Intensity variation

Upper curves, intensity variation across image of tungsten cylinder bombarded by 7-volt electrons. Lower curves, intensity of thermal radiation at $4,200 \mathrm{~A}$

impossible to maintain a polish on the silver electrode in a cæsium discharge, due perhaps to the heating by the large electron currents used.

\section{DISCUSSION}

\section{RADIATION FROM A THIN LAYER}

It is possible to explain the trend of the variation of intensity with angle of emission by assuming that the radiation is emitted by a thin 
layer under the metal surface (radiating uniformly in all directions) and is weakened by reflection losses on being transmitted through the surface. The intensity $I_{\theta}$ emitted at angle $\theta$ is then given by the relation-

$$
I_{\theta}=\text { constant } \times\left(1-R_{\theta}\right) / \cos \theta
$$

$R_{\theta}$ is taken to be the reflection coefficient for plane waves incident upon the surface at angle $\theta$. The factor $1 / \cos \theta$ arises from the fact that a given area of the image receives radiation from an area of the surface larger in the ratio $1 / \cos \theta$.

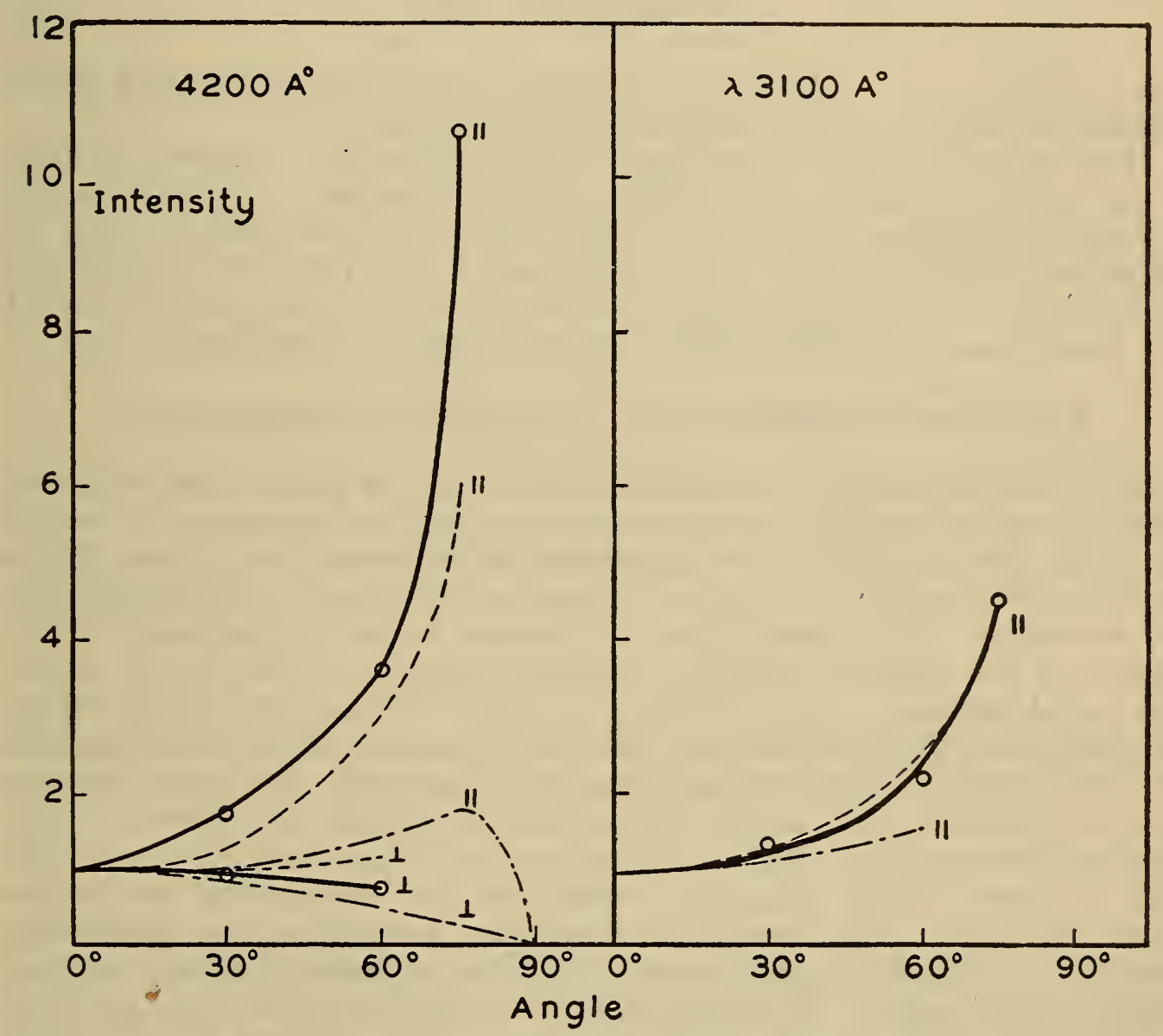

FIGURE 2.-Variation of intensity with angle of emission of radiation from platinum bombarded by 7 -volt electrons, full line

- - Plot of $\left(1-R_{\theta}\right) / \cos \theta$

- - Variation of intensity with angle deduced from the lamellar absorption of platinum.

The dashed lines of Figure 2 are plotted from equation (1). The reflecting powers are computed from the approximation formula given by Drude ${ }^{4}$ using the optical constants of platinum given by the International Critical Tables.

The explanation given above is reasonable only if the concepts of rays and the laws of reflection of plane waves can be applied to waves which have a radius of curvature of a few angstroms at the reflecting surface. The latter distance is in all probability the depth from which the radiation is emitted. It is difficult to see, therefore, why the simple considerations used should be correct. A more reasonable

\footnotetext{
1 Drude, Ann, d. Phys., vol, 35, p. 523, 1888.
} 
method of estimating the variation of surface emissivity with angle will be given below (relation of surface emission to "lamellar absorption").

\section{THE INTENSITY VARIATION OF THERMAL RADIATION WITH ANGLE OF EMISSION}

The intensity of the thermal radiation emitted by a square centimeter is proportional to $\left(1-R_{\theta}\right) \cos \theta 0^{5}$ The apparent intensity observed at an angle $\theta$ is therefore proportional to $\left(1-R_{\theta}\right)$ or

$$
I=\text { constant }\left(1-R_{\theta}\right)
$$

This relation can be derived by assuming an indefinitely thick layer instead of the thin layer used to obtain equation (1).

It is evident that a measurement of the intensity across the image of the cylinder raised to incandescence enables one to estimate the change of reflecting power with angle, $R_{\theta}$. This was done in order to estimate the reliability of the measurements. The reflection coefficients relative to normal incidence were about 18 per cent lower at $60^{\circ}$ than those computed from optical constants of platinum.

\section{SURFACE EMISSION AND " LAMELLAR ABSORPTION"}

Fry ${ }^{6}$ has discussed the absorption of energy by metal surface layers from incident plane light waves using classical electromagnetic theory. He finds the absorption to be somewhat different from that of the metal in bulk and uses the term "lamellar absorption" to describe the phenomenon. The absorption is supposed to be proportional to the square of the electric intensity (radiation energy density) just under the metal surface. Just outside the metal surface the electric intensity is given by the vector sum of electric vectors of the incident and reflected wave. The electric intensity just inside the metal surface can be obtained from the fact that the tangential component of the electric force and the product of the normal component by the amplitude squared of the index of refraction are continuous across the metal surface. The lamellar absorption computed on this basis should by Kirchhoff's law be equal to the emissivity of the surface layers of the metal. Fry gives a curve showing the variation of the lamellar absorption with angle of incidence of the light for platinum at $4,359 \mathrm{~A}$. The curve should also give directly the variation of intensity of emission with angle and should be directly comparable ${ }^{7}$ with the curves of Figure 2. The variation of intensity with angle obtained in this way is not much different from that of thermal radiation, and is therefore not adequate to explain the variation of intensity observed in the present work. If the intensity for normal emission is unity, then at $60^{\circ}$ one obtains from the lamellar absorption curves 1.4 for parallel light; for "perpendicular" light, 0.50 . The

\footnotetext{
5 R. H. Fowler, Statistical Mechanics, p. 493.

6 T. C. Fry, J. Opt. Soc. Am., vol. 22, p. 307, 1932.

7 Consider a metal surface in equilibrium with radiation at some temperature and let a suitably defined beam of the radiation with unit cross section area and energy flux $I$ be incident upon a metal surface at angle $\theta$. Let $a I$ be the energy absorbed from the beam by a surface layer of definite thickness. Also let $e I$ be the energy emitted by the surface layer in the direction of the incident beam and from an area of the surface equal to $1 / \cos \theta$. Equilibrium between the black body radiation and the surface layer demands that $e$ and $a$ be equal. $a$ as a function of angle can be obtained directly from Fry's work. The units of $e$, radiation from an area proportional to $1 / \cos \theta$, are those used in Figure 2. The curves of Fry for the variation of $a$ with angle for platinum at $4,359 \mathrm{~A}$ and those of Figure 2 are therefore directly comparable.
} 
observed values at 4,200 $\mathrm{A}$ are 3.6 and 0.77 . For comparison the variation of emission with angle deduced from the lamellar absorption is also plotted in Figure 2.

\section{RELATION TO THE INVERSE PHOTO-ELECTRIC EFFECT}

Another explanation of the angle intensity relations is possible if the phenomenon is correlated with the inverse vectorial photoelectric effect. Relations between the angle variations of the direct and inverse effects can be obtained by considering the conditions to be fulfilled in order that a metal surface be in thermal equilibrium with black body radiation and the thermal electrons in the space about the metal.

The following is a brief discussion based on the "principle of detailed balance." Let $I d w$ be the energy of black body radiation of given frequency passing through unit area, $s$ (perpendicular to the beam), and striking the metal surface at angle $\theta$. The directions of the "rays" in the beam are supposed to be in the solid angle $d w$. Define $P_{\theta}$ such that $I P_{\theta} d w$ is the energy absorbed from the beam that is used in ejecting photo-electrons from the metal.

Also let $J$ be the thermal electron current ${ }^{8}$ striking unit area of the metal surface and define $i_{\theta} d w$ such that $i_{\theta} J d w$ is the energy emitted by the metal in the solid angle $d w$ as a result of bombardment by the thermal electrons. Then $J i_{\theta} d w / \cos \theta$ is the radiant energy from the metal passing through the unit area $s$. This latter quantity must by the principle of detailed balance equal the energy absorbed photo-electrically from the beam, or

$$
J i_{\theta} d w / \cos \theta=P_{\theta} I d w
$$

$i_{\theta} / \cos \theta$ is the apparent intensity of radiation from the surface observed at angle $\theta$; that is, the quantity measured; if we call it $i_{\theta}^{\prime}$

$$
i_{\theta}^{\prime}=c P_{\theta}
$$

where $c$ is independent of angle.

This latter equation implies that the ordinates of Figures 1 and 2 represent also the variation of photo-electric current with angle, $P_{\theta}$.

The experimental data for the variation of photo-electric current with angle of incidence of the light is rather meager, at least for metals other than the alkalies. ${ }^{9}$ For the alkalies the current with the electric vector parallel to the plane of incidence may be from 2 to 10 times greater at $60^{\circ}$ than at normal incidence. For the other direction of polarization the current is usually proportional to the incident energy minus that reflected $\left(1-R_{\theta}\right)$. For metals other than the alkalies the increase with angle for "parallel" radiation is much slower. The variation can be expressed by the relations

$$
\begin{gathered}
P_{\theta}\|=\alpha\|\left(1-R_{\theta} \|\right) \\
P_{\theta} \perp=\alpha \perp\left(1-R_{\theta} \perp\right)
\end{gathered}
$$

If $\alpha$ is made unity at normal incidence then $\alpha \|$ at $60^{\circ}$ varies from 1 to 2 or $3 ; \alpha \perp$ remains closely unity for all angles. It may be mentioned

$8 \mathrm{Po}$ should be thought of as referring to the ejection of electrons of some definite velocity and direction and $J$ as the current of electrons having the same velocity and opposite direction.

$\checkmark$ Photo-electric Phenomena, A. L. Hughes and L. A. Dubridge, McGraw-Hill, 1932. 
that $\alpha \|>1$ is the criterion for the existence of the vectorial photoelectric effect.

If one uses the computed reflecting powers of platinum one obtains from Figure 2 a value for $\alpha \|$ at $60^{\circ}$ and 4,200 A of 2.4, for $\alpha \perp, 1.3$; at $3,100 \mathrm{~A} \alpha \|$ at $60^{\circ}$ is 1.6 . These values are not inconsistent with the general trend of the photo-electric measurements.

Ives ${ }^{10}$ has successfully explained many phases of the vectorial photo-electric effect for the alkalies by assuming that the photoelectric current is proportional to the lamellar absorbing power of the surface layer emitting the photo-electrons. If this is true for platinum then one would predict for platinum at $4,359 \mathrm{~A}$ only a small vectorial effect; that is, $\alpha \|>1$ and $\alpha \perp$ not much different from unity. The variation of surface emissivity with angle would then be identical with that predicted from the lamellar absorption and would be in conflict with the measurements.

It may be remarked that, if one could use the energy density just above the metal surface instead of that inside as a measure of the absorption or photo-electric current, then one would obtain a very large vectorial effect. If one assumes a gradual transition in energy density between the interior and exterior of the metal, one could explain almost any degree of vectorial effect for the absorption of the first few atom layers. The work of Ives suggests, however, that the discontinuity in energy density is very sharp, occurring within less than an atom diameter.

\section{RELATION TO THE VARIATION OF INTENSITY WITH ANGLE OF THE CONTINUOUS X-RAYS}

The theory of surface emission given in footnote 7 assumes that the individual atoms radiate uniformly in all directions. ${ }^{11}$ The discrepancy between theory and observation may, therefore, be explained by assuming the emitting centers to radiate anisotropically. Such anisotropic radiation is observed for the continuous $\mathrm{X}$ rays and is predicted for the radiation from a stream of slow electrons impinging on protons. ${ }^{12}$ From analogy to both of these cases the radiation should be most intense when observed in a direction perpendicular to the electron velocity and should be polarized with the electric vector parallel to the direction of motion of the electrons. This is in the right direction to explain the discrepancy since it is found that the "parallel radiation" is much more intense at oblique angles than would be predicted for radiation emitted isotropically. This statement is clear if it is remembered that in the present measurements the electric vector of the "parallel" radiation becomes nearly parallel to the electron velocity at large angles of emission. It is difficult, however, to say quantitatively what should be expected on this view.

In this connection it is of interest to recall the work of Lillienfeld and Rother ${ }^{13}$ on the polarization of the visible radiation produced by high speed cathode rays striking a metal target. They observed the polarization for various directions of incidence of the cathode rays and for angles of emission varying from $45^{\circ}$ to a very oblique angle.

${ }^{10}$ H. E. Ives and H. B. Briggs, Phys. Rev., vol. 40, p. 802, 1932.

11 The absorption of a single volume element of the metal is supposed to depend only on the magnitude of the electric vector. This implies that the emission of the element should be independent of direction, its modification by refraction producing the observed angle variation.

12 A. Sommerfeld, Ann. d. Phys., vol. 11, 257, 1931.

13 Lillienfeld and Rother, Phys. Żeit., vol. 21, p. 360, 1920. 
The radiation was found to be always completely polarized and in the direction to be expected if it were emitted by a classical oscillator vibrating perpendicular to the metal surface. The results can not be explained by a purely optical theory. They suggest, however, that the radiation is produced by the electrons being accelerated through the "work function layer" of the metal. Since such an acceleration is normal to the metal surface, classical electromagnetic theory predicts precisely the type of polarization of the emitted radiation that is observed.

\section{SUMMARY}

The variation of intensity with angle for both directions of polarization is given approximately by the relation $I(\theta)=(1-R \theta) / \cos \theta$. The equation is obtained by plausibility arguments and has perhaps not a very sound logical basis.

Angle variation based on sounder reasoning (by use of lamellar absorption theory and Kirchhoff's law) fails to explain the facts. The discrepancy may be due either to the incorrectness of the theory or to the fact that the radiation is initially emitted anisotropically as are the continuous $X$ rays.

Washington, August 3, 1932. 